\title{
Early- versus Late-Onset Alzheimer Disease: Long-Term Functional Outcomes, Nursing Home Placement, and Risk Factors for Rate of Progression
}

\author{
Carina Wattmo Åsa K. Wallin \\ Clinical Memory Research Unit, Department of Clinical Sciences, Malmö, Lund University, \\ Malmö, Sweden
}

\section{Keywords}

Activities of daily living - Cholinesterase inhibitors - Early-onset Alzheimer disease ·

Late-onset Alzheimer disease · Nursing home placement · Longitudinal study

\section{Abstract}

Background/Aims: Whether age at onset influences functional deterioration in Alzheimer disease (AD) is unclear. We, therefore, investigated risk factors for progression in activities of daily living (ADL) and nursing home placement (NHP) in cholinesterase inhibitor (ChEI)-treated patients with early-onset AD (EOAD) versus late-onset AD (LOAD). Methods: This 3-year, prospective, observational, multicenter study included 1,017 participants with mild-to-moderate AD; 143 had EOAD (onset $<65$ years) and 874 LOAD (onset $\geq 65$ years). Possible sociodemographic and clinical factors that could affect functional outcome and NHP were analyzed using mixed-effects models and logistic regression, respectively. Results: Younger individuals exhibited longer illness duration before AD diagnosis, whereas 6-month functional response to ChEI therapy, 3-year changes in ADL capacities, time from diagnosis to NHP, and survival time in nursing homes were similar between the groups. In LOAD, a higher ChEI dose, no antidepressant use, and lower education level were protective factors for slower instrumental ADL (IADL) decline. In EOAD, antihypertensives/cardiac therapy implied faster IADL progression but lower risk of NHP. Conclusion: This study highlights the clinical importance of an earlier diagnosis and treatment initiation and the need for functional evaluations in EOAD. Despite the age differences between EOAD and LOAD, a similar need for nursing homes was observed. 
Wattmo and Wallin: Early- versus Late-Onset Alzheimer Disease: Long-Term Functional Outcomes, Nursing Home Placement, and Risk Factors for Rate of Progression

\section{Introduction}

Early-onset Alzheimer disease (EOAD) is defined as Alzheimer disease (AD) with an onset before the age of 65 years. In addition, these younger patients may have more hereditary and aggressive forms of AD [1]. The consequences of being diagnosed early with a disease that implies progressive decline of cognitive abilities and activities of daily living (ADL) performance, as well as changes in personality and behavioral disturbances, are enormous. Many of the affected individuals and their spouses are still in the workforce, have an active social life, and might even have children living at home. Hence, EOAD caregivers are often faced with other commitments, such as working and taking care of the family and household [2]. Deterioration in ADL is a major distressing aspect of dementia for the family members, and the severity of functional impairment is considered to be the predominant critical factor behind nursing home placement (NHP) [3, 4]. In addition, individuals with EOAD lose 15-18 years of average life expectancy [5]. Despite these facts, there have been fewer reports of ADL compared with cognitive outcomes in earlier studies of EOAD [6, 7].

Currently, the predominant therapy for mild-to-moderate AD is cholinesterase inhibitors (ChEIs). Placebo-controlled clinical trials with a duration of up to 1 year have shown that ChEIs are effective in slowing functional decline [8,9]. Extension studies $[10,11]$ and observational studies of ChEI treatment $[12,13]$ have suggested that the effect of ChEIs on ADL may last even longer. However, because all these AD studies have included participants without regard to age at onset, no previous ChEI studies have focused on EOAD or aspects of therapy (e.g., type of drug and dose) that might alter functional progression in younger patients.

Very few studies of AD have investigated potential predictors (e.g., age) of short-term therapeutic response to ChEIs and long-term outcome in ADL. Our group found that both functional response to ChEIs [14] and performance after 3 years of treatment [12] were better in younger than in older individuals. Another study did not observe a longitudinal association between age and impairment in ADL after ChEI or ChEI + memantine therapy [15]. Inconsistency among untreated participants with $\mathrm{AD}$ has also been reported; one study found a faster rate of functional deterioration in EOAD [16], whereas another study showed a similar progression among ages [17]. Older age is a common predictor of early NHP in dementia [18, 19]; a recent study found that the time from symptom onset to NHP was more than double among the younger dementia cohort [20]. However, when adjusting for many sociodemographic and clinical characteristics, age was not a significant risk factor that influenced time to NHP in our study using multivariate models [4]. In EOAD, possible predictors (e.g., years of education and comorbidity) that might affect the results and lead to inconsistent observations have not been considered in most studies [6].

The aims of this study were to describe and compare functional long-term outcomes and NHP between patients with EOAD and late-onset Alzheimer disease (LOAD) in a routine clinical setting, and to identify the sociodemographic and clinical factors, including aspects of ChEI therapy, that affect the rate of progression in the respective groups.

\section{Material and Methods}

\section{Study and Participants}

The Swedish Alzheimer Treatment Study (SATS) is a 3-year, prospective, observational, multicenter study that was established to assess the effectiveness of long-term ChEI treatment (donepezil, rivastigmine, and galantamine) in a routine clinical setting. The SATS has been reported at length and various findings were published in a number of articles [4, 5, 12, 14, 21]. In total, 1,258 participants with $A D$ were enrolled from 14 memory clinics located across 
Sweden. All 1,021 individuals who showed a baseline Mini-Mental State Examination (MMSE) [22] score ranging from 10 to 26, and whose age was known at the estimated onset of AD (4 had missing data) were included in the current study. Of these, 143 patients were defined as having EOAD (onset of $\mathrm{AD}<65$ years) and 874 as having LOAD (onset of $\mathrm{AD} \geq 65$ years); thus, 1,017 participants were enrolled.

Considered for inclusion in the SATS were outpatients aged 40 years and older who met the criteria for the clinical diagnosis of dementia, as defined by the Diagnostic and Statistical Manual of Mental Disorders, 4th edition (DSM-IV) [23], and for possible or probable AD, according to the criteria of the National Institute of Neurological and Communicative Disorders and Stroke and the Alzheimer's Disease and Related Disorders Association (NINCDS-ADRDA) [24]. All individuals were diagnosed by physicians specialized in dementia disorders, who also estimated the age at onset based on an interview with the caregiver (usually the spouse or an adult child) concerning their observations of early symptoms of AD. Moreover, the selected patients had to be community dwelling with or without home help services at the time of AD diagnosis, have a reliable caregiver, and be assessable with the MMSE scale at the start of ChEI therapy (baseline). The exclusion criteria were not fulfilling the diagnostic criteria for AD, already taking active ChEI treatment or contraindications to ChEIs. After inclusion in the SATS and the baseline evaluations, the participants were prescribed ChEI therapy as part of the ordinary Swedish health care system, with respect to the approved product labeling. The study is observational and the choice of drug agent and dosage was left up to the dementia specialist's discretion and professional judgment. The ChEI dose was documented after 2 months of treatment and then semiannually after baseline. Medications other than ChEIs were recorded at baseline and allowed during the study, with the exception of memantine. If the patient stopped taking the ChEI, or if memantine was added, the individual was excluded from the SATS at that time point. The date of and reason for any withdrawal from the study were documented.

\section{Ethics Approval and Consent to Participate}

All procedures performed in studies involving the SATS participants were in accordance with the Helsinki Declaration. The SATS protocol and the present analysis of data from the study reported in this paper were submitted to and approved by the Regional Ethical Review Board, Lund University, Sweden (No. 2014/658, December 9, 2014). Written informed consent was obtained from all patients included in the SATS. If an individual was not able to provide consent for him/herself, consent was obtained from his/her closest relative.

\section{Outcome Measures}

The SATS patients were assessed in a structured, follow-up program over 3 years that investigated cognition, global, instrumental, and basic ADL capacities at the start of ChEI therapy, after 2 months (MMSE and global rating only), and every 6 months. Cognitive ability was evaluated using the MMSE, with scores ranging from 0 to 30 (a lower score indicating worse cognitive status) and the Alzheimer's Disease Assessment Scale-cognitive subscale (ADAS-cog) [25], with a total range of 0-70 (a higher score indicating worse cognitive status).

The Instrumental Activities of Daily Living (IADL) scale [26] consists of 8 different items: ability to use the telephone, shopping, food preparation, housekeeping, laundry, mode of transportation, responsibility for own medications, and ability to handle finances. Each item was scored from 1 (no impairment) to 3-5 (severe impairment), which allowed a total range of 8-31 points. The Physical Self-Maintenance Scale (PSMS) [26] consists of 6 different items: toilet, feeding, dressing, grooming, physical ambulation, and bathing. Each item was scored from 1 (no impairment) to 5 (severe impairment), which yielded a total range of 6-30 points. Nurses trained to care for people with dementia assessed the ADL performance based on 
Wattmo and Wallin: Early- versus Late-Onset Alzheimer Disease: Long-Term Functional

interviews with the caregiver. To facilitate the comparison of rates on the ADAS-cog, IADL, and PSMS scores, changes in score were converted to positive values, which were indicative of improvement, and negative values, which were indicative of decline.

NHP was defined as the date of permanent admission to a licensed skilled nursing facility with 24-h care; rehabilitative or respite care was not included. If hospitalization occurred before nursing home entry, the date of hospital admission was used. Using the 12-digit personal identity number assigned to each resident of Sweden, we determined whether each participant in the SATS was still alive on December 31, 2015 with the help of the Swedish population register (Swedish Tax Agency). If not, the date of death was recorded.

\section{Statistical Analyses}

The IBM Statistical Package for the Social Sciences (SPSS) for Windows (version 22.0; IBM Corporation, Armonk, NY, USA) was used to perform the statistical analyses. The level of significance was defined as $p<0.05$ if not otherwise noted, and all tests were 2-tailed. Observed-case analyses were used to avoid overestimation of the treatment effect by imputing better previous outcome scores in a longitudinal study of a progressively advancing disease. Parametric tests were used because of the large sample size and the approximately normally distributed continuous possible predictors. Independent-sample $t$ tests were performed to compare the differences between the means obtained for 2 groups, such as EOAD and LOAD, and $\chi^{2}$ tests were computed to analyze categorical variables.

Mixed, linear and nonlinear, fixed and random coefficient regression models with the patient as a hierarchical variable (i.e., to allow correlation within subjects) were used. The mixed-models method also considers differences in the number of visits available for each individual and unequal time intervals between the follow-up evaluations, which are common statistical limitations found in long-term studies. Those who discontinued the SATS contributed information during the time of participation; thus, we took into account the trajectories of all patients.

Time was defined as the exact number of months between the start of ChEI therapy and each assessment, thereby using all data points at the actual time intervals. To adjust for baseline differences, the initial instrumental or basic ADL scores for each participant and their interaction with linear and quadratic terms for time in the study (to enable a nonlinear rate of change in the models) were included as fixed effects, i.e., time in months (and time in months ${ }^{2}$ ) $\times$ IADL (or PSMS) baseline score. Thus, the dependent variables were the functional scores assigned at the second and subsequent visits for each patient; that is, the mixed-effects models do not intend to predict the scores at the start of ChEI treatment. The random terms were an intercept and time in months, with a variance components covariance matrix. Several potential sociodemographic and clinical characteristics were included as fixed effects in the models, such as sex, age at the initiation of ChEI therapy, clinician's estimated duration of AD, years of education, carrier of the apolipoprotein E (APOE) $\varepsilon 4$ allele (no/yes), solitary living (no/yes), cognitive status, number of medications at baseline, and specific concomitant medications (no/yes for each group) including antihypertensives/cardiac therapy, antidiabetics, asthma medication, thyroid therapy, lipid-lowering agents, estrogens, nonsteroidal antiinflammatory drugs/acetylsalicylic acid, antidepressants, antipsychotics, and anxiolytics/ sedatives/hypnotics. The effect of ChEIs was investigated using the type of drug (coded as a set of dummy variables) and dosages. The ChEI dose could vary during the treatment period for an individual patient and between patients; hence, the mean dose used during the entire follow-up period was calculated for each participant. Regarding the noncompleters, the mean dose used during the individual's time of participation in the SATS was calculated. To obtain a similar metric for the percentage of maximum dosage for each of the $3 \mathrm{ChEIs}$, the mean dose was divided by the maximum recommended dose for each drug, that is, $10 \mathrm{mg}$ for donepezil, 
$12 \mathrm{mg}$ for rivastigmine (oral administration), and $24 \mathrm{mg}$ for galantamine. The term "ChEI agent $\times$ dose" was also included in the models. Finally, some possible interactions (sex, age, or education) with functional capacity at baseline or with time in the study were included. Nonsignificant variables $(p>0.05)$ were removed in a backward stepwise manner. The hierarchical principle was applied in the mixed-effects models; variables that appeared in significant interactions were not considered for removal.

Binary logistic regression analyses using the backward likelihood ratio method were used to predict NHP during the study for patients with EOAD and LOAD. The HosmerLemeshow goodness-of-fit test result was $p>0.05$, indicating a good fit of the models to the data. The aforementioned potential characteristics were used as independent variables together with the ADAS-cog, IADL, and PSMS mean rates of deterioration per month.

\section{Results}

Sociodemographic and Clinical Characteristics according to Age at Onset of AD

The 1,017 SATS participants were divided into 2 groups according to the age at onset of AD: EOAD (<65 years: $n=143,14 \%$ ) and LOAD ( $\geq 65$ years: $n=874,86 \%)$. The sociodemographic and clinical characteristics of the groups are presented in Table 1. At the initiation of ChEI treatment (baseline), a smaller percentage of the younger individuals were living alone $\left(\chi^{2}(1)=13.66 ; p<0.001\right)$. Moreover, lower proportions of antihypertensives/cardiac therapy $\left(\chi^{2}(1)=30.25 ; p<0.001\right)$, nonsteroidal anti-inflammatory drugs/acetylsalicylic acid $\left(\chi^{2}(1)=\right.$ 29.64; $p<0.001)$, and anxiolytics/sedatives/hypnotics $\left(\chi^{2}(1)=14.16 ; p<0.001\right)$ were found in the EOAD versus the LOAD cohort. The younger patients also had a longer mean duration of $\mathrm{AD}\left(t_{1,015}=4.36 ; p<0.001\right)$, and more years of education $\left(t_{1,015}=2.93 ; p=0.004\right)$ than the older individuals. At baseline, the patients with EOAD exhibited less functional impairment according to the IADL score $\left(t_{993}=-4.92 ; p<0.001\right)$ and PSMS score $\left(t_{994}=-7.14 ; p<0.001\right)$, and took fewer concomitant medications $\left(t_{1,015}=-8.20 ; p<0.001\right)$ compared with the LOAD group.

\section{Comparison of Longitudinal Outcomes between EOAD and LOAD}

Regarding the IADL score, $51 \%$ of the EOAD and $48 \%$ of the LOAD patients were improved/ unchanged ( $\leq 0$ point change) after 6 months of ChEI treatment $\left(\chi^{2}(1)=0.26 ; p=0.611\right)$. Improvement/no change in PSMS score after 6 months was observed in $79 \%$ of the younger and $73 \%$ of the older individuals in the SATS $\left(\chi^{2}(1)=1.52 ; p=0.217\right)$.

Consistently, no significant difference in disease progression over time between the onset groups was detected when using the IADL or PSMS scales. After 3 years of ChEI therapy, the mean decline (95\% confidence interval [CI]) from baseline did not differ between the EOAD and LOAD cohorts: IADL score, $6.0(4.6-7.4)$ versus $6.6(6.0-7.2)$ points $\left(t_{353}=0.83\right.$; $p=0.406)$; PSMS score, $2.6(1.8-3.5)$ versus $2.9(2.5-3.3)$ points $\left(t_{354}=0.53 ; p=0.598\right)$. The patients with EOAD and LOAD were further divided into APOE genotypes. No significant differences in changes in IADL or basic ADL performance after 3 years of ChEI treatment were found between these groups.

The IADL capacity was already markedly impaired at the start of ChEI therapy, with $40-65 \%$ of the EOAD and 55-75\% of the LOAD patients being dependent on assistance to perform these activities (IADL score, 2-5). The percentage of participants with impairment in the individual IADL items was significantly lower at baseline in the EOAD cohort, with the exception of "ability to handle finances," which did not differ between the onset groups. After 3 years, the IADL performance had deteriorated further; $70-90 \%$ of the remaining patients in both groups could not carry out these tasks independently. Younger individuals showed a 
Table 1. Sociodemographic and clinical characteristics $(n=1,017)$

\begin{tabular}{|c|c|c|c|}
\hline Variable & $\begin{array}{l}\text { Early-onset AD } \\
(n=143,14 \%)\end{array}$ & $\begin{array}{l}\text { Late-onset AD } \\
(n=874,86 \%)\end{array}$ & $p$ \\
\hline Female sex, $n / \%$ & $82 / 57$ & $568 / 65$ & 0.091 \\
\hline APOE genotype $(n=996), n / \%$ & & & $<0.001$ \\
\hline No $\varepsilon 4$ alleles & $36 / 25$ & $284 / 33$ & \\
\hline $1 \varepsilon 4$ allele & $66 / 46$ & $459 / 54$ & \\
\hline $2 \varepsilon 4$ alleles & $41 / 29$ & $110 / 13$ & \\
\hline Solitary living at baseline, $n / \%$ & $30 / 21$ & $322 / 37$ & $<0.001$ \\
\hline Antihypertensives/cardiac therapy, $n / \%$ & $28 / 20$ & $384 / 44$ & $<0.001$ \\
\hline Antidiabetics, $n / \%$ & $5 / 3$ & $45 / 5$ & 0.397 \\
\hline Asthma medication, $n / \%$ & $9 / 6$ & $34 / 4$ & 0.185 \\
\hline Thyroid therapy, $n / \%$ & $9 / 6$ & $76 / 9$ & 0.336 \\
\hline Lipid-lowering agents, $n / \%$ & $16 / 11$ & $101 / 12$ & 0.898 \\
\hline Estrogens, $n / \%$ & $9 / 6$ & $60 / 7$ & 0.801 \\
\hline NSAIDs/acetylsalicylic acid, $n / \%$ & $15 / 10$ & $288 / 33$ & $<0.001$ \\
\hline Antidepressants, $n / \%$ & $41 / 29$ & $215 / 25$ & 0.298 \\
\hline Antipsychotics, $n / \%$ & $2 / 1$ & $43 / 5$ & 0.058 \\
\hline Anxiolytics/sedatives/hypnotics, $n / \%$ & $6 / 4$ & $141 / 16$ & $<0.001$ \\
\hline Estimated age at onset (mean $\pm S D$ ), years & $58.6 \pm 4.7$ & $74.4 \pm 4.9$ & $<0.001$ \\
\hline Estimated duration of AD at baseline (mean \pm SD), years & $4.1 \pm 3.4$ & $2.9 \pm 1.7$ & $<0.001$ \\
\hline Age at first assessment (mean \pm SD), years & $62.7 \pm 5.4$ & $77.3 \pm 4.7$ & $<0.001$ \\
\hline Education (mean \pm SD), years & $10.1 \pm 2.8$ & $9.3 \pm 2.5$ & 0.004 \\
\hline MMSE score at baseline (mean \pm SD) & $21.4 \pm 3.8$ & $21.4 \pm 3.7$ & 0.987 \\
\hline ADAS-cog score $(0-70)$ at baseline $($ mean \pm SD) & $19.5 \pm 9.6$ & $21.0 \pm 8.8$ & 0.074 \\
\hline IADL score at baseline (mean $\pm S D)$ & $13.9 \pm 5.3$ & $16.3 \pm 5.4$ & $<0.001$ \\
\hline PSMS score at baseline (mean \pm SD) & $6.7 \pm 1.2$ & $7.6 \pm 2.4$ & $<0.001$ \\
\hline Number of concomitant medications at baseline (mean \pm SD) & $1.8 \pm 1.7$ & $3.1 \pm 2.5$ & $<0.001$ \\
\hline Length in the SATS (mean \pm SD), months & $25.1 \pm 12.4$ & $22.9 \pm 13.1$ & 0.065 \\
\hline \multicolumn{4}{|c|}{ Dose of ChEI during the follow-up period (mean \pm SD), mg } \\
\hline Donepezil $(n=516)^{\mathrm{a}}$ & $7.4 \pm 1.9(40 \%)$ & $6.8 \pm 1.7(52 \%)$ & 0.020 \\
\hline Rivastigmine $(n=211)^{\mathrm{a}}$ & $6.6 \pm 2.3(26.5 \%)$ & $6.0 \pm 2.1(20 \%)$ & 0.100 \\
\hline Galantamine $(n=290)^{\mathrm{a}}$ & $15.8 \pm 3.6(33.5 \%)$ & $15.1 \pm 3.8(28 \%)$ & 0.184 \\
\hline
\end{tabular}

AD, Alzheimer disease; ADAS-cog, Alzheimer's Disease Assessment Scale-cognitive subscale; APOE, apolipoprotein E; ChEI, cholinesterase inhibitor; IADL, Instrumental Activities of Daily Living scale; MMSE, Mini-Mental State Examination; NSAIDs, nonsteroidal anti-inflammatory drugs; PSMS, Physical SelfMaintenance Scale; SATS, Swedish Alzheimer Treatment Study.

a Percentage of patients in each group that received the specific ChEI agent in parentheses $\left(\chi^{2}\right.$ test; $p=$ $0.017)$.

faster decline in "ability to use the telephone," "shopping," "food preparation," and "housekeeping." However, the participants with LOAD were still significantly worse in "laundry," "mode of transportation," and "responsibility for own medications" (Fig. 1a).

Regarding basic ADL, the majority of patients were able to manage themselves independently at baseline, with the exception of physical ambulation $(>50 \%$ of the individuals with LOAD needed some assistance; PSMS score, 2-5). A significantly larger percentage of the older cohort was impaired in the ADL items "toilet," "physical ambulation," and "bathing." After 3 years, $35-55 \%$ of the remaining participants in the SATS needed assistance in performing the basic ADL tasks "dressing," "grooming," and "bathing," while $60 \%$ of the EOAD and $75 \%$ of the LOAD patients required help with "physical ambulation" ( $p=0.030)$. The younger individuals demonstrated a more rapid deterioration in "toilet" and "bathing" (Fig. 1b). 
Wattmo and Wallin: Early- versus Late-Onset Alzheimer Disease: Long-Term Functional

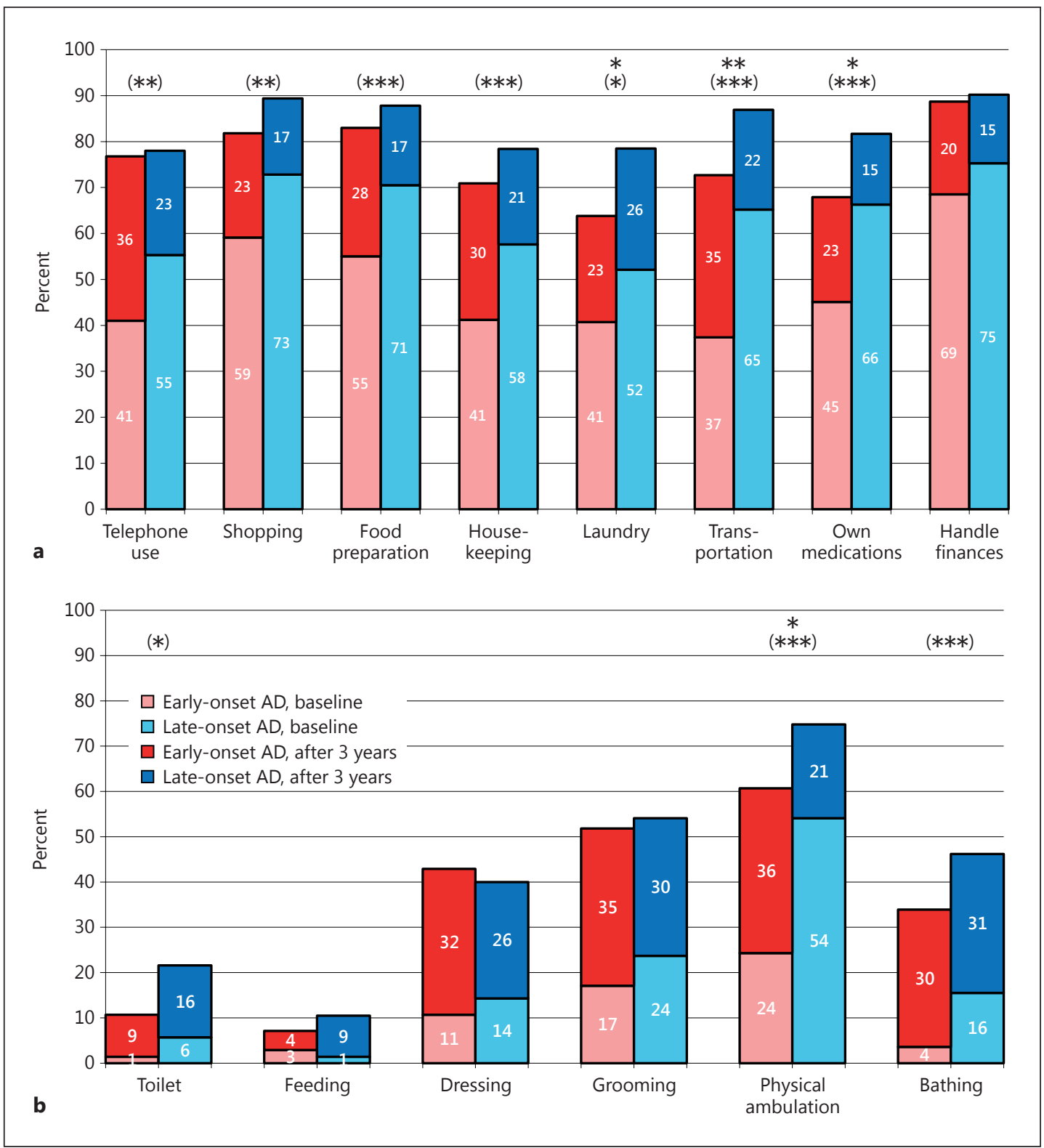

Fig. 1. The proportion of SATS patients who needed help to carry out IADL and basic ADL tasks by onset group. a The percentage of patients with EOAD and LOAD who needed help to carry out IADL tasks (IADL score, 2-5) at the start of ChEI therapy (baseline, in parentheses) and after 3 years (*0.01 $\leq p<0.05$, ** $\left.0.001 \leq p<0.01,{ }^{* * *} p<0.001\right)$. b The percentage of patients who needed help to carry out basic ADL tasks (PSMS score, 2-5) at the baseline (in parentheses) and after 3 years according to onset group $(* 0.01 \leq p<$ $\left.0.05, * * 0.001 \leq p<0.01,{ }^{* * *} p<0.001\right)$. ADL, activities of daily living; ChEI, cholinesterase inhibitor; EOAD, early-onset Alzheimer disease; IADL, Instrumental Activities of Daily Living scale; LOAD, late-onset Alzheimer disease; PSMS, Physical Self-Maintenance Scale; SATS, Swedish Alzheimer Treatment Study.

\section{Predictors of Disease Progression in the Respective Age at Onset Groups}

Only participants with 3 or more evaluations were included in the mixed-effects models to enable analyses of a nonlinear rate of functional change (EOAD, $n=120$, 84\%; LOAD, $n=$ $665,76 \%$ ). The models were performed to identify the sociodemographic and clinical factors that influenced the SATS patients' longitudinal trajectories (EOAD, 524 data points; 
Wattmo and Wallin: Early- versus Late-Onset Alzheimer Disease: Long-Term Functional Outcomes, Nursing Home Placement, and Risk Factors for Rate of Progression

Table 2. Factors affecting the long-term outcome with IADL score as dependent variable according to age at onset

\begin{tabular}{|c|c|c|c|c|c|c|}
\hline & \multicolumn{3}{|c|}{ Early-onset AD } & \multicolumn{3}{|c|}{ Late-onset $\mathrm{AD}$} \\
\hline & $\beta$ & $95 \% \mathrm{CI}$ & $p$ & $\beta$ & $95 \% \mathrm{CI}$ & $p$ \\
\hline \multicolumn{7}{|l|}{ Fixed terms ${ }^{\mathrm{a}}$} \\
\hline Intercept & 2.021 & $0.157,3.885$ & 0.034 & -2.542 & $-4.922,-0.162$ & 0.036 \\
\hline Time in months from baseline & 0.234 & $0.198,0.270$ & $<0.001$ & 0.206 & $0.130,0.281$ & $<0.001$ \\
\hline IADL score at baseline & 0.884 & $0.769,0.999$ & $<0.001$ & 1.560 & $1.313,1.806$ & $<0.001$ \\
\hline IADL score at baseline ${ }^{2}$ & & & ns & -0.021 & $-0.028,-0.014$ & $<0.001$ \\
\hline Time in months $\times$ IADL score at baseline & & & ns & -0.002 & $-0.005,0.002$ & 0.335 \\
\hline Time in months ${ }^{2} \times$ IADL score at baseline & & & ns & -0.00007 & $-0.00012,-0.00002$ & 0.006 \\
\hline \multicolumn{7}{|l|}{ Background variables } \\
\hline Solitary living (no $=0$, yes $=1$ ) & 1.250 & $0.050,2.450$ & 0.041 & & & ns \\
\hline \multicolumn{7}{|l|}{ Antihypertensives/cardiac therapy } \\
\hline$($ no $=0$, yes $=1)$ & 1.551 & $0.338,2.763$ & 0.013 & & & ns \\
\hline Antidepressants $($ no $=0$, yes $=1$ ) & & & ns & 0.570 & $0.076,1.065$ & 0.024 \\
\hline Education, years & & & ns & -0.055 & $-0.153,0.043$ & 0.274 \\
\hline Time in months $\times$ education in years & & & ns & 0.008 & $0.002,0.014$ & 0.007 \\
\hline ADAS-cog score at baseline & 0.075 & $0.014,0.136$ & 0.016 & 0.057 & $0.028,0.086$ & $<0.001$ \\
\hline ChEI dose $\mathrm{e}^{\mathrm{b}}$ & & & ns & -0.016 & $-0.028,-0.004$ & 0.010 \\
\hline \multicolumn{7}{|l|}{ Random terms (variance) } \\
\hline Intercept & 3.999 & $2.506,6.382$ & $<0.001$ & 4.787 & $4.002,5.725$ & $<0.001$ \\
\hline Time in months & 0.026 & $0.018,0.037$ & $<0.001$ & 0.019 & $0.016,0.023$ & $<0.001$ \\
\hline
\end{tabular}

LOAD, 2,836 data points). The percentages of variance that accounted for the dependent variable, regarding all fixed predictors, were $65.3 \%$ for IADL and $16.8 \%$ for PSMS in the EOAD group, and $63.3 \%$ for IADL and $43.0 \%$ for PSMS in the LOAD group. This indicates a good to very good fit of the models ( $p<0.001$ for all models); however, the degree of explanation was lower for the PSMS model in the EOAD cohort. The mixed-effects models, significant predictors, and unstandardized $\beta$ coefficients with $95 \% \mathrm{CI}$ are presented in Tables 2 and 3.

Individuals with better cognitive status at the initiation of ChEI treatment in both onset groups exhibited a more favorable long-term outcome in IADL capacity. In EOAD patients, the risk factors associated with faster IADL decline were solitary living and use of antihypertensives/cardiac therapy, while more years of education showed a trend toward significance ( $p=0.061$ ). In LOAD patients, the use of antidepressants, a higher level of education, and a lower dose of ChEI independently predicted worse IADL performance. Furthermore, there was an interaction effect between years of education and time in study; that is, more years of education implied increased IADL impairment over time. Regarding basic ADL, use of anxiolytics/sedatives/hypnotics implied slower functional progression in the EOAD cohort; however, few younger patients used this medication $(n=6)$. Lower cognitive ability at baseline predicted a faster deterioration of basic ADL among the individuals with LOAD. 
Wattmo and Wallin: Early- versus Late-Onset Alzheimer Disease: Long-Term Functional

Outcomes, Nursing Home Placement, and Risk Factors for Rate of Progression

Table 3. Factors affecting the long-term outcome with PSMS score as dependent variable according to age at onset

\begin{tabular}{|c|c|c|c|c|c|c|}
\hline & \multicolumn{3}{|c|}{ Early-onset AD } & \multicolumn{3}{|c|}{ Late-onset AD } \\
\hline & $\beta$ & $95 \% \mathrm{CI}$ & $p$ & $\beta$ & $95 \% \mathrm{CI}$ & $p$ \\
\hline \multicolumn{7}{|l|}{ Fixed terms $s^{\mathrm{a}}$} \\
\hline Intercept & 2.243 & $0.316,4.169$ & 0.023 & 0.437 & $-0.138,1.011$ & 0.136 \\
\hline Time in months from baseline & -0.095 & $-0.251,0.061$ & 0.229 & 0.057 & $0.012,0.103$ & 0.013 \\
\hline PSMS score at baseline & 0.379 & $0.136,0.622$ & 0.003 & 0.812 & $0.735,0.890$ & $<0.001$ \\
\hline Time in months $\times$ PSMS score at baseline & 0.032 & $0.008,0.055$ & 0.008 & 0.005 & $-0.002,0.011$ & 0.179 \\
\hline Time in months ${ }^{2} \times$ PSMS score at baseline & & & ns & 0.00010 & $0.00002,0.00019$ & 0.017 \\
\hline \multicolumn{7}{|l|}{ Background variables } \\
\hline \multicolumn{7}{|l|}{ Anxiolytics/sedatives/hypnotics } \\
\hline$($ no $=0$, yes $=1)$ & -1.488 & $-2.762,-0.213$ & 0.023 & & & ns \\
\hline ADAS-cog score at baseline & & & ns & 0.030 & $0.015,0.046$ & $<0.001$ \\
\hline \multicolumn{7}{|l|}{ Random terms (variance) } \\
\hline Intercept & 0.599 & $0.219,1.643$ & 0.052 & 0.777 & $0.517,1.169$ & $<0.001$ \\
\hline Time in months & 0.016 & $0.011,0.022$ & $<0.001$ & 0.013 & $0.011,0.016$ & $<0.001$ \\
\hline
\end{tabular}

Sex, number of apolipoprotein E $\varepsilon 4$ alleles, solitary living, age at baseline, duration of AD, years of education, number of medications and specific concomitant medications with the exception of anxiolytics/sedatives/hypnotics, ChEI dose, and the variable comparing the ChEI agents were not significant predictors in the models. $\beta$ values were unstandardized and are expressed per 1 unit increase for continuous variables and for the condition present in dichotomous variables. AD, Alzheimer disease; ADAS-cog, Alzheimer's Disease Assessment Scale-cognitive subscale; ChEI, cholinesterase inhibitors; CI, confidence interval; ns, not significant; PSMS, Physical SelfMaintenance Scale.

${ }^{\mathrm{a}}$ Variance accounted for (all fixed terms): early-onset AD, 16.8\% $(p<0.001)$; late-onset AD, 43.0\% $(p<0.001)$.

\section{End Points in EOAD versus $L O A D$}

Overall, 86 patients (60\%) with EOAD and $556(64 \%)$ with LOAD did not complete the 3 -year SATS $\left(\chi^{2}(1)=0.64 ; p=0.425\right)$. The reasons for the dropout were admission to nursing homes $(n=131,13 \%)$, initiation of concomitant memantine therapy $(n=81,8 \%)$, side effects $(n=80,8 \%)$, withdrawal of informed consent $(n=55,5 \%)$, poor effect/deterioration $(n=54$, $5 \%)$, compliance problems ( $n=49,5 \%)$, somatic disease assumed to be unrelated to $\operatorname{AD~}(n=$ $30,3 \%)$, switching to another ChEI agent $(n=21,2 \%)$, and other reasons $(n=60,6 \%)$. No differences between the age at onset groups were observed for the aforementioned reasons. Among the younger versus the older patients, switching to another study was more common (13 [9\%] vs. $\left.21[2 \%], \chi^{2}(1)=17.01 ; p<0.001\right)$, whereas death as a dropout reason was less frequent $\left(1[1 \%]\right.$ vs. $\left.46[5 \%], \chi^{2}(1)=5.81 ; p=0.016\right)$.

During the SATS, 26 patients (18\%) with EOAD and $205(23 \%)$ with LOAD $\left(\chi^{2}(1)=1.66\right.$; $p=0.196)$ were admitted to nursing homes. Figure 2a presents a Kaplan-Meier graph of the time distribution from baseline to NHP for the 2 groups (log-rank test, $p=0.064$ ). The respective mean time $(95 \% \mathrm{CI})$ from estimated onset of AD to NHP for the younger and older cohorts was $8.4(6.6-10.2)$ versus $4.6(4.3-4.9)$ years $\left(t_{229}=7.58 ; p<0.001\right)$. However, the time from the start of ChEI treatment to institutionalization for participants with EOAD and LOAD (22.3 [18.7-25.8] vs. 19.3 [18.0-20.7] months; $\left.t_{229}=1.42 ; p=0.156\right)$ and the mean survival time in nursing homes (5.1 [3.8-6.5] vs. 4.1 [3.7-4.5] years; $t_{221}=1.65 ; p=0.100$ ) were similar between both onset groups. For all individuals, risk factors for NHP were solitary living, worse IADL capacity at baseline, and a faster rate of IADL decline during the study. In the EOAD cohort, more years of education and use of antihypertensives/cardiac therapy were predictors of a lower risk of institutionalization (Table 4).

At the end of the 3-year SATS, 8 participants (6\%) with EOAD and 127 (15\%) with LOAD had died $\left(\chi^{2}(1)=8.53 ; p=0.002\right)$; after up to 18 years of follow-up, $115(80 \%)$ and $797(91 \%)$ 
Fig. 2. Time from baseline to the end points NHP and death. a Kaplan-Meier graph of the distribution of time from the start of ChEI therapy (approximately time of AD diagnosis) to NHP for the SATS participants with early- versus late-onset $\mathrm{AD}$. Using the log-rank test, no difference was found between the groups ( $p=0.064)$. b Kaplan-Meier graph of the distribution of time from the start of ChEI therapy to death according to onset of $\mathrm{AD}$. A log-rank test showed a shorter life expectancy for patients with late-onset $\mathrm{AD}$ $(p<0.001)$. AD, Alzheimer disease; ChEI, cholinesterase inhibitor; NHP, nursing home placement; SATS, Swedish Alzheimer Treatment Study.

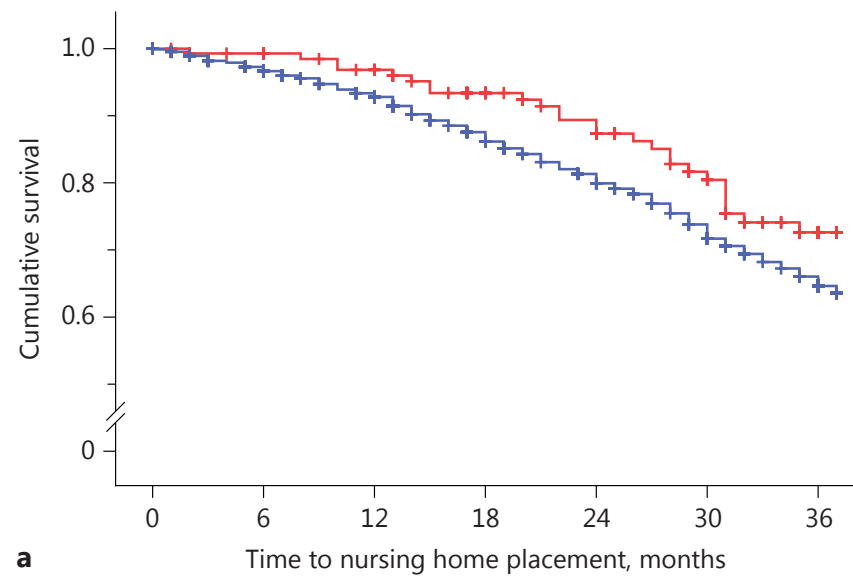

a

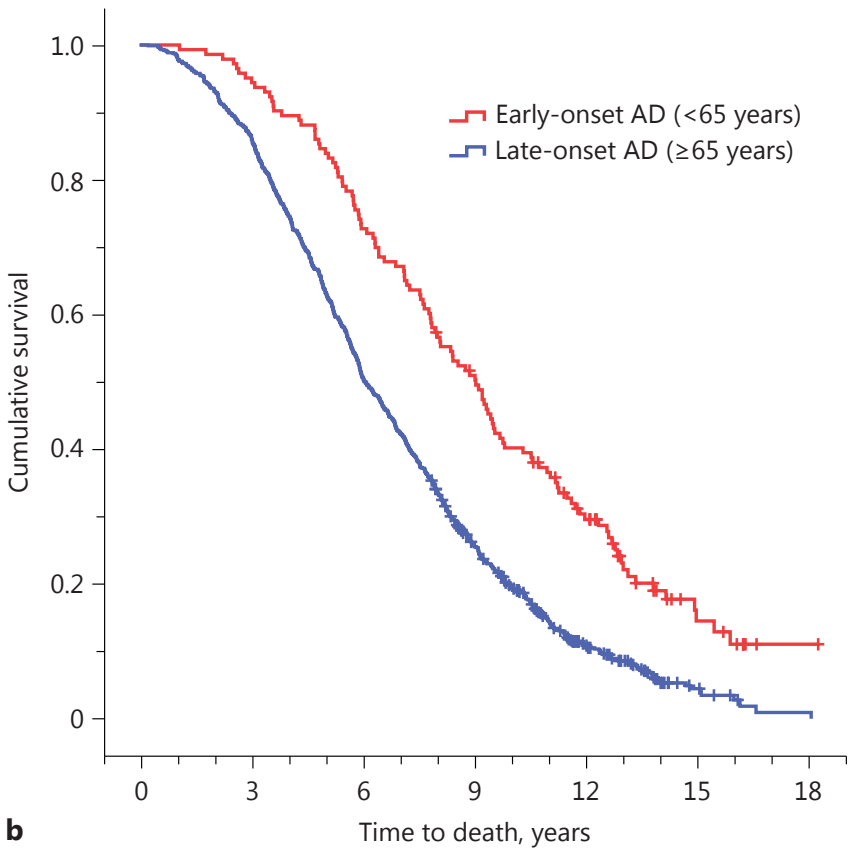

of the patients $\left(\chi^{2}(1)=15.40 ; p<0.001\right)$ had died, respectively. Figure $2 \mathrm{~b}$ presents the KaplanMeier graph of time distribution from the initiation of ChEI treatment to death for patients with EOAD and LOAD (log-rank test, $p<0.001)$. The mean $(95 \% \mathrm{CI})$ survival time from baseline was $8.0(7.4-8.6)$ versus $6.2(6.0-6.4)$ years $\left(t_{910}=5.72 ; p<0.001\right)$, and age at death was $71.1(70.0-72.1)$ versus $83.6(83.3-84.0)$ years $\left(t_{910}=-24.07 ; p<0.001\right)$ for patients with EOAD and LOAD, respectively. Users of antihypertensives/cardiac therapy showed a shorter life expectancy from baseline on average than nonusers (5.8 [5.5-6.1] vs. 6.8 [6.6-7.1] years; $\left.t_{910}=4.92 ; p<0.001\right)$. No differences in mortality were detected for the variables solitary living or level of education. 
Table 4. Binary logistic regression analysis of nursing home placement during the study according to age at onset

\begin{tabular}{|c|c|c|c|c|}
\hline & \multicolumn{2}{|c|}{ Early-onset AD $(n=143)$} & \multicolumn{2}{|c|}{ Late-Onset $\mathrm{AD}(n=874)$} \\
\hline & OR $(95 \% \mathrm{CI})$ & $p$ & OR $(95 \% \mathrm{CI})$ & $p$ \\
\hline Solitary living (no $=0$, yes $=1)^{\mathrm{a}}$ & $8.24(2.19-31.04)$ & 0.002 & $2.75(1.93-3.93)$ & $<0.001$ \\
\hline Antihypertensives /cardiac therapy $($ no $=0$, yes $=1)$ & $0.08(0.01-0.95)$ & 0.045 & & ns \\
\hline Education, years & $0.73(0.56-0.93)$ & 0.013 & & ns \\
\hline IADL score at baseline & $1.27(1.11-1.45)$ & $<0.001$ & $1.12(1.08-1.16)$ & $<0.001$ \\
\hline IADL score, rate of change per month & $0.01(0.0005-0.14)$ & 0.001 & $0.28(0.15-0.51)$ & $<0.001$ \\
\hline
\end{tabular}

Correctly classified: early-onset $\mathrm{AD}, 87.2 \%$; late-onset $\mathrm{AD}, 77.2 \%$. ORs are expressed per 1 unit increase for continuous variables and for the condition present for categorized variables. Sex, number of apolipoprotein E $\varepsilon 4$ alleles, age at baseline, duration of AD, ADAS-cog and PSMS scores at baseline or their rates of change per month, number of medications and specific concomitant medications with the exception of antihypertensives/cardiac therapy, ChEI dose, and the variable comparing the ChEI agents were not significant factors in the models. AD, Alzheimer disease; ADAS-cog, Alzheimer's Disease Assessment Scalecognitive subscale; CI, confidence interval; IADL, Instrumental Activities of Daily Living scale; ns, not significant; OR, odds ratio; PSMS, Physical Self-Maintenance Scale.

${ }^{a}$ The interaction effect of sex with solitary living was significant for the late-onset AD group $(p<0.016)$. Male living with family member was the reference category. Male living alone, OR 5.12 (95\% CI, 2.29-11.47); female living with family, OR 1.83 (1.12-2.99); female living alone, OR 3.72 (2.30-6.00). Because of the low number of younger males living alone ( $n=9)$, it was not possible to include the interaction term in the early-onset AD model.

\section{Discussion}

In the SATS, performed in a routine clinical setting, the functional 6-month response to ChEI treatment and the longitudinal outcomes over 3 years were similar between the onset groups; however, a somewhat faster deterioration in some IADL tasks was found in EOAD patients. Better cognitive ability at baseline predicted a more positive functional performance over time for all participants. A higher dose of ChEI and fewer years of education were protective factors for slower IADL progression in the LOAD cohort, whereas a higher level of education in EOAD patients implied less institutionalization. Users of antihypertensives/ cardiac therapy among the younger individuals exhibited a more rapid rate of IADL decline but lower risk of NHP. Solitary living in patients with EOAD and use of antidepressants in LOAD were also factors related to worse IADL capacities. The time to institutionalization and survival time in nursing homes were similar between those admitted in the onset groups, although the mean life span after AD diagnosis was $~ 2$ years longer among the younger individuals.

The EOAD cohort in the current study demonstrated significantly more years of education, longer illness duration, and less functional impairment but similar cognitive ability at the start of ChEI treatment compared with the older patients. More years of education was associated with faster IADL deterioration in LOAD patients, while a trend toward significance was observed for participants with EOAD. The slightly increased rapid progression in some IADL tasks among the younger group in this study might reflect their higher education and better "brain reserve capacity." These findings support the cognitive reserve hypothesis in which people with more years of education are expected to have a more advanced disease with higher burden of AD pathology at the time of diagnosis [27, 28]. A higher level of education was also a protective factor for NHP among our EOAD patients. One explanation might be the aforementioned worse decline as well as an earlier death shown in highly educated individuals with AD [5]. Nursing homes are publicly funded in Sweden and not dependent on the resident's financial situation or insurance coverage; thus, socioeconomic status is not expected to influence NHP [29]. In agreement with our observations, a review reported an extended 
Wattmo and Wallin: Early- versus Late-Onset Alzheimer Disease: Long-Term Functional Outcomes, Nursing Home Placement, and Risk Factors for Rate of Progression

time to NHP for dementia patients with more years of education [30], and a recent study also found that younger people with dementia (who had higher education) were cared for at home longer than older individuals [20]. A younger, well-educated person with AD might have a larger social support network, be more assertive for home help services, and be less willing to be admitted to a nursing home, most likely among substantially older residents. Therefore, a larger proportion of EOAD patients might be cared for in their own home for the survival time they have remaining.

Risk factors for worse IADL performance in the SATS were solitary living among the younger and use of antidepressants among the older participants. A smaller percentage of EOAD compared with LOAD patients were living alone, which could be expected because of more advanced age and thus higher mortality among spouses of older individuals. An AD diagnosis at a younger age might result in more unexpected loss of independence and diminishing social activities, and an increased need for support to perform daily tasks. In addition, possible effects of solitary living, such as social isolation, apathy, and symptoms of depression, could precipitate the course of the disease. Apathy was suggested to be the most prevalent neuropsychiatric symptom in EOAD [31] and it has been described as a predictor of faster functional deterioration and a marker of a more aggressive dementia [32]. Moreover, apathy was a significant predictor of NHP in early-onset but not late-onset dementia [20]. Depression might be related to cerebrovascular pathology, which is more common among older people [33]. A more rapid progression in IADL has been observed particularly in milder AD patients with depression, which might depend on recognition of their decreased capacities and loss of independence, as well as reduced initiative and motivation [34]. Decrease in motor activity and lack of responsiveness were the strongest predictors of functional impairment in a 2-year study of depression in AD [35]. In agreement with results from the Swedish Dementia Registry [36], no difference in the frequency of antidepressants between the onset groups was detected in the SATS. However, antidepressant medications might not be effective for all individuals with $\mathrm{AD}$ [37]. Our findings suggest that depression in LOAD, even if treated pharmaceutically, could be associated with worse outcome in IADL. Furthermore, the impact of depression on IADL decline in this study indicates that older participants might be in a milder stage of AD because of a reduced cognitive reserve.

In the present study, use of antihypertensives/cardiac therapy was also a risk factor for faster IADL deterioration in EOAD. As expected, the use of these medications was less common in the younger SATS cohort in agreement with other studies [36]. Hypertension has been identified as a possible risk factor for EOAD [38]. However, the strength of the relationship between cardiovascular changes and AD may be modifiable by exercise and physical function, which might be more pertinent in younger people [39]. One explanation for our observation is that the EOAD patients who used antihypertensives/cardiac therapy might have a lower general health status and are less physically active, which might lead to decreased performance in IADL.

Short-term functional response to ChEI treatment did not differ between the onset groups in this study, whereas higher doses of ChEIs were related to a slower rate of IADL decline in LOAD. The association between a higher ChEI dose and slower functional progression has been reported in the entire mild-to-moderate SATS cohort [12]. The lack of association between IADL capacities over time and ChEI dose among the younger participants might depend on their higher cognitive reserve, resulting in more advanced disease and AD pathology at diagnosis and start of treatment despite a lower functional impairment measured by rating scales. In agreement with our observations, milder disease at the initiation of ChEI therapy and optimal doses can lead to a better ability to maintain cognition and ADL for a longer time $[12,40]$. Taken together, this underlines the importance of early diagnosis in EOAD and thus initiation of antidementia drugs at a milder stage of the disease. 
Functional outcome was similar between the age at onset groups after 3 years in the current study and stresses the clinical importance of functional assessments also among younger patients. Consistent with this finding, time from AD diagnosis to NHP ( 20 months) and survival time in nursing homes (4-5 years) did not differ between the age groups. Previous studies have shown inconsistent results concerning whether the EOAD cohort progresses faster $[16,17]$. A recent Dutch study of early- versus late-onset dementia in general [20] reported almost identical times on average from disease onset to institutionalization (earlyonset dementia 8.9 years vs. late-onset dementia 4.2 years) to those reported in the SATS (8.4 vs. 4.6 years). The time between symptom onset and inclusion (time of diagnosis was not addressed) in the Dutch study was more than double among the young participants with dementia ( 7.4 vs. 3.3 years). In our study, the mean time between estimated onset and diagnosis was significantly longer in the EOAD group (4.1 vs. 2.9 years). These observations demonstrate a delay in diagnosis among the younger individuals with dementia. Information about expected time to NHP and length of stay in nursing homes is important knowledge for community-based services.

The strengths of the 3-year SATS are the prospective, well-organized, 6-month evaluations after the start of ChEIs in a large cohort of continuously treated "real-life" AD patients with concomitant disorders and medications from memory clinics in different parts of Sweden. Because of publicly financed health care, the participants are expected to be representative of the general population. One limitation is that the SATS was not placebo controlled because of ethical aspects, or randomized according to ChEI drug type, as in other observational AD studies over longer periods. Another weakness of this study, similar to earlier publications of age at onset [16], is that the clinician's estimation of the onset of AD symptoms was dependent on the thoroughness and validity of information obtained from the caregiver.

Very few long-term studies have analyzed the associations between EOAD and LOAD, level of education, functional capacities, concomitant medications, and NHP; additional studies are therefore warranted. The potential effect of ChEIs, predictors of disease progression, and factors that might affect the usage of community-based services, and time to NHP in different age groups need further investigation. In most countries, knowledge of younger AD patients and their families' special needs and support, and care facilities specially designed for this group are lacking. Furthermore, service utilization, respite care, and nursing homes directed toward patients with EOAD are needed to avoid severely affected individuals being cared for among considerably older residents.

In conclusion, a comparison of risk factors for functional impairment and time to NHP between participants with EOAD and LOAD was reported in this observational, longitudinal study. After 3 years, overall functional deterioration was similar between the onset groups; however, some IADL tasks tended to show faster decline among younger patients over this period. This finding underlines the clinical importance of functional assessments in EOAD. Solitary living and use of antihypertensives/cardiac therapy were independent risk factors for worse IADL outcome in the younger cohort, which might depend on social isolation and apathy, and lower general health status and less physical activity, respectively. The use of antidepressants in LOAD might also precipitate progression in IADL; this observation indicates that psychiatric symptoms are a risk factor for worse functional prognosis. A higher mean dose of ChEI was related to better IADL performance in LOAD but not in EOAD, which might depend on a more advanced disease in younger individuals because of their higher cognitive reserve. In addition, longer illness duration before AD diagnosis was shown among the younger patients. These findings stress the importance of early detection of EOAD and initiation of treatment to increase the effects of antidementia therapy. Time from diagnosis to NHP ( 20 months) and survival time in nursing homes ( $4-5$ years) did not differ between the age groups, which is important information for community-based services. 


\section{Acknowledgment}

This work was supported by a postdoctoral scholarship from the Swedish Brain Foundation to C.W.; by grants from Greta och Johan Kocks stiftelse (Fromma Foundation) and SUS (Skånes universitetssjukhus) stiftelser och donationer (Skåne University Hospital Foundations and Donations) in Sweden to C.W.; and by Stiftelsen Gamla Tjänarinnor (Foundation of Old Servants) to Å.K.W. The sponsors had no involvement in the study design, in the collection, analysis, and interpretation of data, in the writing of the report, or in the decision to submit the manuscript.

We wish to thank all the SATS patients and their relatives for their cooperation in this study. The authors are grateful to the staff from all of the different centers that took part in the management of the participants and provided administrative support to the study.

\section{Disclosure Statement}

The authors have no conflicts of interest to report.

\section{Author Contributions}

C.W. participated in the SATS, supervised the data collection, was responsible for the statistical design, and conducted the statistical analyses, interpreted the results, and drafted the manuscript. Å.K.W. participated in the study, supervised the data collection, assisted in the analysis and interpretation of the data, and revised the manuscript critically. Both authors read and approved the final manuscript.

\section{References}

1 Wu L, Rosa-Neto P, Hsiung GY, Sadovnick AD, Masellis M, Black SE, Jia J, Gauthier S: Early-onset familial Alzheimer's disease (EOFAD). Can J Neurol Sci 2012;39:436-445.

-2 Bakker C, de Vugt ME, van Vliet D, Verhey FR, Pijnenburg YA, Vernooij-Dassen MJ, Koopmans RT: The use of formal and informal care in early onset dementia: results from the NeedYD study. Am J Geriatr Psychiatry 2013;21:37-45.

-3 Hebert R, Dubois MF, Wolfson C, Chambers L, Cohen C: Factors associated with long-term institutionalization of older people with dementia: data from the Canadian Study of Health and Aging. J Gerontol A Biol Sci Med Sci 2001;56:M693-M699.

4 Wattmo C, Wallin AK, Londos E, Minthon L: Risk factors for nursing home placement in Alzheimer's disease: a longitudinal study of cognition, ADL, service utilization, and cholinesterase inhibitor treatment. Gerontologist 2011;51:17-27.

5 Wattmo C, Londos E, Minthon L: Risk factors that affect life expectancy in Alzheimer's disease: a 15-year follow-up. Dement Geriatr Cogn Disord 2014;38:286-299.

6 Stanley K, Walker Z: Do patients with young onset Alzheimer's disease deteriorate faster than those with late onset Alzheimer's disease? A review of the literature. Int Psychogeriatr 2014;26:1945-1953.

7 Gronning H, Rahmani A, Gyllenborg J, Dessau RB, Hogh P: Does Alzheimer's disease with early onset progress faster than with late onset? A case-control study of clinical progression and cerebrospinal fluid biomarkers. Dement Geriatr Cogn Disord 2012;33:111-117.

-8 Mohs RC, Doody RS, Morris JC, Ieni JR, Rogers SL, Perdomo CA, Pratt RD: A 1-year, placebo-controlled preservation of function survival study of donepezil in AD patients. Neurology 2001;57:481-488.

-9 Potkin SG, Anand R, Hartman R, Veach J, Grossberg G: Impact of Alzheimer's disease and rivastigmine treatment on activities of daily living over the course of mild to moderately severe disease. Prog Neuropsychopharmacol Biol Psychiatry 2002;26:713-720.

10 Winblad B, Wimo A, Engedal K, Soininen H, Verhey F, Waldemar G, Wetterholm AL, Haglund A, Zhang R, Schindler R: 3-year study of donepezil therapy in Alzheimer's disease: effects of early and continuous therapy. Dement Geriatr Cogn Disord 2006;21:353-363. 
Wattmo and Wallin: Early- versus Late-Onset Alzheimer Disease: Long-Term Functional

11 Pirttila T, Wilcock G, Truyen L, Damaraju CV: Long-term efficacy and safety of galantamine in patients with mild-to-moderate Alzheimer's disease: multicenter trial. Eur J Neurol 2004;11:734-741.

12 Wattmo C, Wallin AK, Londos E, Minthon L: Long-term outcome and prediction models of activities of daily living in Alzheimer disease with cholinesterase inhibitor treatment. Alzheimer Dis Assoc Disord 2011;25: 63-72.

13 Behl P, Lanctot KL, Streiner DL, Black SE: The effect of cholinesterase inhibitors on decline in multiple functional domains in Alzheimer's disease: a two-year observational study in the Sunnybrook dementia cohort. Int Psychogeriatr 2008;20:1141-1159.

14 Wattmo C, Wallin AK, Minthon L: Functional response to cholinesterase inhibitor therapy in a naturalistic Alzheimer's disease cohort. BMC Neurol 2012;12:134.

15 Atri A, Shaughnessy LW, Locascio JJ, Growdon JH: Long-term course and effectiveness of combination therapy in Alzheimer disease. Alzheimer Dis Assoc Disord 2008;22:209-221.

-16 Jacobs D, Sano M, Marder K, Bell K, Bylsma F, Lafleche G, Albert M, Brandt J, Stern Y: Age at onset of Alzheimer's disease: relation to pattern of cognitive dysfunction and rate of decline. Neurology 1994;44:1215-1220.

17 Koss E, Edland S, Fillenbaum G, Mohs R, Clark C, Galasko D, Morris JC: Clinical and neuropsychological differences between patients with earlier and later onset of Alzheimer's disease: a CERAD analysis, Part XII. Neurology 1996;46:136-141.

18 Hatoum HT, Thomas SK, Lin SJ, Lane R, Bullock R: Predicting time to nursing home placement based on activities of daily living scores - a modelling analysis using data on Alzheimer's disease patients receiving rivastigmine or donepezil. J Med Econ 2009;12:98-103.

19 Heyman A, Peterson B, Fillenbaum G, Pieper C: Predictors of time to institutionalization of patients with Alzheimer's disease: the CERAD experience, part XVII. Neurology 1997;48:1304-1309.

20 Bakker C, de Vugt ME, van Vliet D, Verhey FR, Pijnenburg YA, Vernooij-Dassen MJ, Koopmans RT: Predictors of the time to institutionalization in young- versus late-onset dementia: results from the Needs in Young Onset Dementia (NeedYD) study. J Am Med Dir Assoc 2013;14:248-253.

21 Wallin AK, Andreasen N, Eriksson S, Batsman S, Nasman B, Ekdahl A, Kilander L, Grut M, Ryden M, Wallin A, Jonsson M, Olofsson H, Londos E, Wattmo C, Eriksdotter Jonhagen M, Minthon L: Donepezil in Alzheimer's disease: what to expect after 3 years of treatment in a routine clinical setting. Dement Geriatr Cogn Disord 2007;23:150-160.

-22 Folstein MF, Folstein SE, McHugh PR: "Mini-mental state". A practical method for grading the cognitive state of patients for the clinician. J Psychiatr Res 1975;12:189-198.

23 Frances A; American Psychiatric Association: Diagnostic and Statistical Manual of Mental Disorders: DSM-IV: Prepared by the Task Force on DSM-IV, ed 4. Washington, American Psychiatric Association, 1994.

24 McKhann G, Drachman D, Folstein M, Katzman R, Price D, Stadlan EM: Clinical diagnosis of Alzheimer's disease: report of the NINCDS-ADRDA Work Group under the auspices of Department of Health and Human Services Task Force on Alzheimer's Disease. Neurology 1984;34:939-944.

-25 Rosen WG, Mohs RC, Davis KL: A new rating scale for Alzheimer's disease. Am J Psychiatry 1984;141:13561364.

26 Lawton MP, Brody EM: Assessment of older people: self-maintaining and instrumental activities of daily living. Gerontologist 1969;9:179-186.

27 Stern Y, Albert S, Tang MX, Tsai WY: Rate of memory decline in AD is related to education and occupation: cognitive reserve? Neurology 1999;53:1942-1947.

28 Bennett DA, Wilson RS, Schneider JA, Evans DA, Mendes de Leon CF, Arnold SE, Barnes LL, Bienias JL: Education modifies the relation of AD pathology to level of cognitive function in older persons. Neurology 2003;60: 1909-1915.

29 Lagergren M: The systems of care for frail elderly persons: the case of Sweden. Aging Clin Exp Res 2002;14: 252-257.

-30 Luppa M, Luck T, Brahler E, Konig HH, Riedel-Heller SG: Prediction of institutionalisation in dementia. A systematic review. Dement Geriatr Cogn Disord 2008;26:65-78.

-31 van Vliet D, de Vugt ME, Aalten P, Bakker C, Pijnenburg YA, Vernooij-Dassen MJ, Koopmans RT, Verhey FR: Prevalence of neuropsychiatric symptoms in young-onset compared to late-onset Alzheimer's disease - part 1: findings of the two-year longitudinal NeedYD-study. Dement Geriatr Cogn Disord 2012;34:319-327.

-32 Starkstein SE, Jorge R, Mizrahi R, Robinson RG: A prospective longitudinal study of apathy in Alzheimer's disease. J Neurol Neurosurg Psychiatry 2006;77:8-11.

-33 O’Brien J, Desmond P, Ames D, Schweitzer I, Harrigan S, Tress B: A magnetic resonance imaging study of white matter lesions in depression and Alzheimer's disease. Br J Psychiatry 1996;168:477-485.

-34 Fitz AG, Teri L: Depression, cognition, and functional ability in patients with Alzheimer's disease. J Am Geriatr Soc 1994;42:186-191.

-35 Haupt M, Kurz A, Greifenhagen A: Depression in Alzheimer's disease: phenomenological features and association with severity and progression of cognitive and functional impairment. Int J Geriatr Psychiatry 1995;10: 469-476.

-36 Eriksson H, Fereshtehnejad SM, Falahati F, Farahmand B, Religa D, Eriksdotter M: Differences in routine clinical practice between early and late onset Alzheimer's disease: data from the Swedish Dementia Registry (SveDem). J Alzheimers Dis 2014;41:411-419. 
Wattmo and Wallin: Early- versus Late-Onset Alzheimer Disease: Long-Term Functional Outcomes, Nursing Home Placement, and Risk Factors for Rate of Progression

-37 Banerjee S, Hellier J, Dewey M, Romeo R, Ballard C, Baldwin R, Bentham P, Fox C, Holmes C, Katona C, Knapp M, Lawton C, Lindesay J, Livingston G, McCrae N, Moniz-Cook E, Murray J, Nurock S, Orrell M, O’Brien J, Poppe M, Thomas A, Walwyn R, Wilson K, Burns A: Sertraline or mirtazapine for depression in dementia (HTASADD): a randomised, multicentre, double-blind, placebo-controlled trial. Lancet 2011;378:403-411.

38 Atkins ER, Bulsara MK, Panegyres PK: Cerebrovascular risk factors in early-onset dementia. J Neurol Neurosurg Psychiatry 2012;83:666-667.

39 Purnell C, Gao S, Callahan CM, Hendrie HC: Cardiovascular risk factors and incident Alzheimer disease: a systematic review of the literature. Alzheimer Dis Assoc Disord 2009;23:1-10.

-40 Small G, Bullock R: Defining optimal treatment with cholinesterase inhibitors in Alzheimer's disease. Alzheimers Dement 2011;7:177-184. 